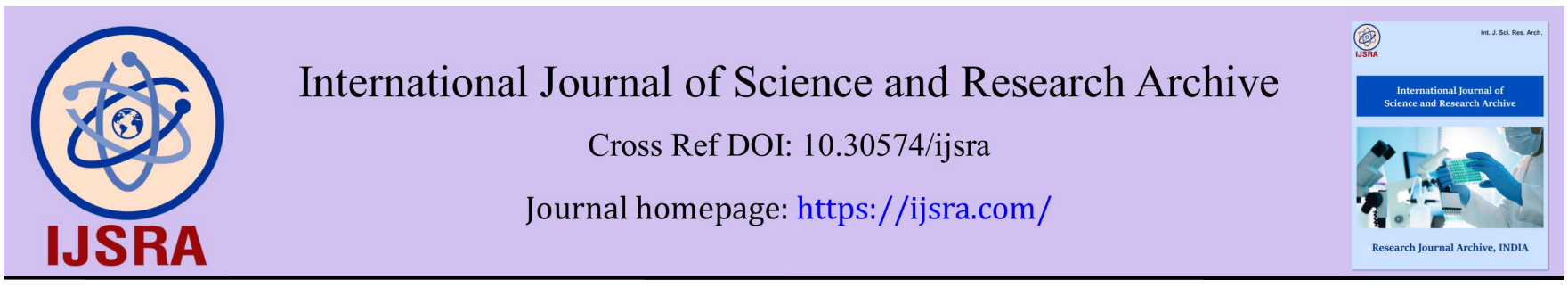

(RESEARCH ARTICLE)

\title{
Acoustic, thermal and optical properties of organic based disodium tartrate salt
}

\author{
M. Sathish ${ }^{1}$, K. Venkataramanan ${ }^{2}$, R. Padmanaban ${ }^{2}$, Helan Ruth ${ }^{3}$, K. Vadivel ${ }^{3,}{ }^{*}$, and G. Ramalingam ${ }^{4}$ \\ ${ }^{1}$ Department of Physics, St. Joseph's College of Arts \& Science, (Autonomous), Cuddalore - 607001. \\ ${ }^{2}$ Department of Physics, SCSVMV University, Kanchipuram-631561. \\ ${ }^{3}$ Department of Physics, Government Arts College, Viruddhachalam 606001. \\ ${ }^{4}$ Department of Nanoscience and Technology, Alagappa University, Karaikudi-630003.
}

International Journal of Science and Research Archive, 2021, 02(01), 012-027

Publication history: Received on 10 January 2021; revised on 18 January 2021; accepted on 20 January 2021

Article DOI: https://doi.org/10.30574/ijsra.2021.2.1.0010

\begin{abstract}
In this work, acoustic, thermal, and optical properties were tested on the different concentrations of the Disodium Tartrate solutions. First, the viscosity studies were analyzed for the Disodium tartrate in the concentration range from $2 \%$ to $20 \%$ with different temperatures $303 \mathrm{~K}, 308 \mathrm{~K}, 313 \mathrm{~K}$, and $318 \mathrm{~K}$. It was noted that the relative viscosity and the activation energy of the prepared compound increase with increases in concentration and decreases with temperature increases. The properties like density and ultrasonic velocity are varied when increases the concentration of the aqueous solutions of Disodium Tartrate. In this study, the values of adiabatic compressibility show an inverse behavior when compared with ultrasonic velocity due to the interaction between solute and solvent molecules. Also observed that the inter-molecular free length is maximum for a lower percentage. The free volume for the compound is maximum at $2 \%$ and a minimum of $20 \%$, since it reduces when the internal pressure increases. It was revealed that the classical absorption coefficient and relaxation time for Disodium Tartrate is minimum for lower percentage and minimum for a higher percentage. The interactions between the solute and solvent are confirmed through the property like specific Acoustical impedance. It was noted that the increase in internal pressure increases the concentration of the compound. The ion-solvent interaction was discussed by the relative association study, thus the values of relative association increases with an increase in concentration. The Rao's and Wada's constant increases linearly in aqueous solutions of Disodium Tartrate for the entire system.
\end{abstract}

Keywords: Ultrasonic; Acoustic; Disodium; Tartrate; Aqueous.

\section{Introduction}

Double salts are salts containing more than one cation or anion. A well-known double salt is an alum containing two cations (Potassium and Aluminum) and a sulfate anion. Other examples are potassium sodium tartrate and bromide. Alums are double sulphates of a monovalent cation and a trivalent cation containing water of crystallization. Double salts when dissolved in water dissociate into simple ions completely ${ }^{[1]}$. Tartaric acid is an organic (carbon-based) compound of the chemical formula $\mathrm{C}_{4} \mathrm{H}_{6} \mathrm{O}_{6}$ and has the official name 2,3-dihydroxybutanedioic acid. In this name, the 2,3- dihydroxy refers to the two $\mathrm{OH}$ - groups on the second and third carbon atoms, and the butane portion of the name refers to a four-carbon molecule. The dioic acid portion communicates the existence of two organic acids $(\mathrm{COOH})$ groups on the molecule. Tartaric acid is found throughout nature, especially in many fruits and in wine ${ }^{[2] .}$

Tartrate salts are often used as food additives due to their ability to act as anti-oxidants. Disodium tartrate dehydrate, additive number (E335), is used as an emulsifier and binding agent in food products such as jam and sugar syrup ${ }^{[3]}$.

\footnotetext{
${ }^{*}$ Corresponding author: K.Vadivel

Department of Physics, Government Arts College, Viruddhachalam 606001.

Copyright (C) 2021 Author(s) retain the copyright of this article. This article is published under the terms of the Creative Commons Attribution Liscense 4.0.
} 
In the present study an attempt has been made to compute the viscosity of aqueous solutions of Di-Sodium tartrate (Molar mass : 230.08) at different concentrations (2\%, 4\%, 6\%, 8\%, 10\%, 12\%, 14\%, 16\%, 18\% \& 20\%), at different temperatures ( $303 \mathrm{~K}, 308 \mathrm{~K}, 313 \mathrm{~K} \& 318 \mathrm{~K})$. From these experimental data, activation energy is calculated and the effect of solvent is analyzed. Density, ultrasonic velocity, and refractive index have been estimated for aqueous solutions of Disodium tartrate at 303K. From these experimental values, various molecular interaction parameters like free volume, internal pressure, viscous relaxation time, inter-atomic free length, etc are calculated and discussed in terms of solute-solvent interactions.

\section{Materials and Methods}

\subsection{Measurement of density}

Measurements of density and viscosity are very useful in the study of tartrate solutions. These values are used to compute some of the parameters such as Molar volume and apparent molecular volume. These parameters are helpful in the investigation of molecular interaction and to tackle the problems relating to the properties of a given system.

Specific gravity bottle which is empty, with its stopper is weighed in the K-ROY make Electronic balance, with an accuracy of $\pm 0.001 \mathrm{gm}$ and its weight up to the third decimal place is noted down as $\mathrm{W}_{1}$. Then the solution whose density is to be measured is filled till the neck of the specific gravity bottle and then the stopper is fitted tight to the neck, the excess solution must spray out of the capillary tube, then the bottle is wiped with tissue paper and then it is weighted on the Electronic balance, and its weight is noted asW 2 .

The density of the solution is determined using the formula:

$$
\begin{gathered}
\rho=\frac{\text { Mass of the solution in } \mathrm{gm}}{\text { Volume of the specific gravity bottle in } c c} \\
\text { Mass of the solution }=W_{2}-W_{1} \mathrm{gm}
\end{gathered}
$$

Where $\mathrm{W}_{2}$ is the weight of the bottle with solution and $\mathrm{W}_{1}$ is the weight of the empty bottle.

$$
\rho=\frac{W_{2}-W_{1}}{\text { Volume of the bottle }} \mathrm{gm} / \mathrm{cc}
$$

The experimental solution was taken into a $10 \mathrm{ml}$ specific gravity bottle. Care was taken to ensure that there were no air bubbles. The mass of the liquid in it was measured by K-ROY make single pan electronic balance with an accuracy of $\pm 0.001 \mathrm{gm}$ and it is measured at $303 \mathrm{~K}$ using an electronically controlled thermostat with an accuracy of $\pm 0.5 \mathrm{~K}$.

\subsection{Abbe refractometer}

Carefully clean and dry both surfaces of the prism body by turning the handle to the right. One glance into the square entrance aperture of the illuminated prism will show that the entire space between the two prisms is evenly filled because air bubbles in the liquid layer reduce the contrast of the borderline. If required take more of the specimen liquid.

Place a refractometer into its position for use and set using pinion head to the beginning of the measuring graduation. Wait a few minutes before measuring so that the temperature becomes constant. In a meantime arrange the mirror in such a manner that the light fills the square aperture of the illuminating prism and the field of view appears bright in the telescope eyepiece.

Turn pinion head slowly towards the higher values until the field of view becomes dark from below. Make color fringe between the two half-fields disappear by turning the knurled knob; set borderline which is now well defined to the point of intersection of the cross - line by further turning of the pinion head. In this position the measured result is read on the $\eta_{D}$ or $\%$ graduation. As the position, of the borderline depends on the refractive index of the specimen and the graduation glass scale is firmly connected with the prism, at this setting of the telescope, the reading microscope immediately indicates with its graduation line the $\eta_{D}$ value required. Thus the refractive of the solution is determined directly. 


\subsection{Tartrate solution preparation}

In the present study, Di-Sodium Tartrate is obtained from Southern India Scientific Company, Trichy (AR Grade). The tartrate solutions were prepared by dissolving the tartrate in double distilled water to give different concentrations of $2 \%, 4 \%, 6 \%, 8 \%, 10 \%, 12 \%, 14 \%, 16 \%, 18 \%$ and $20 \%$.

\section{Results and discussion}

\subsection{Determination of activation energy}

Viscosity studies are performed for an aqueous solution of Di-Sodium tartrate in the concentration range of $2 \%-20 \%$ at different temperatures $303 \mathrm{~K}, 308 \mathrm{~K}, 313 \mathrm{~K}$, and 318K. Figure 1 and Table 1 show the variation of relative viscosity against concentration for an aqueous solution of Di-Sodium tartrate at different temperatures. In this case, the relative viscosity increases with the increase in concentration and decreases with the increase in temperature. For all the systems, the relative viscosity is minimum at lower concentration (2\%) and at a higher concentration range (20\%) it is maximum. It may be due to a more frictional force developed between the layers of the solution.

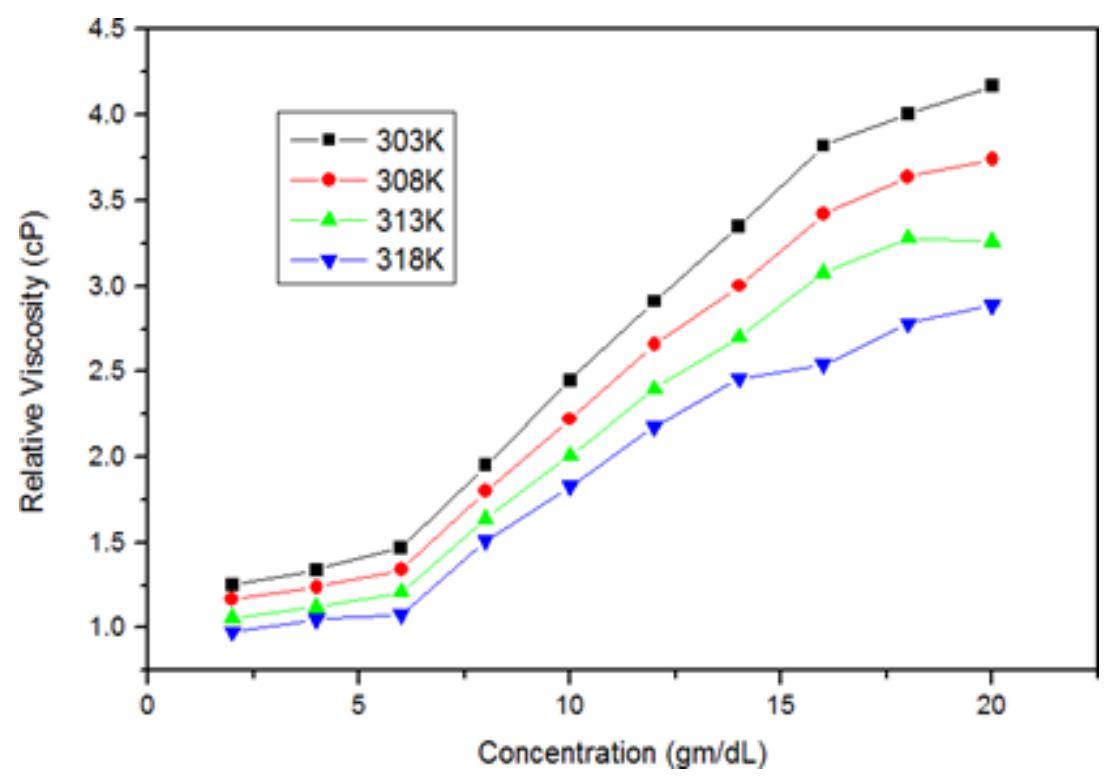

Figure 1 Variation of Relative Viscosity against Concentration

Arrhenius expression is valid for pure solvents and also for dilute polymer solutions.

According to Moore et al [4]

$\eta=A e^{Q / R T}$

Where Q- is the apparent activation energy of flow and A is the pre-exponential term with an activation entropy significance. The logarithm of this equation leads to a straight-line type.

$\ln \eta=\ln A+\left(\frac{Q}{R}\right) \frac{1}{T}$

Figure 2 shows the variation of $\log$ viscosity ( $\ln \eta$ ) against the inverse of temperature $(1 / \mathrm{T})$ for an aqueous solution of Di-Sodium tartrate. The $\ln \eta$ against 1 /T graph shows that the curve fit is a straight line. In all the cases, the ln $\eta-$ value increases with concentration and decreases with a decrease in temperature. 


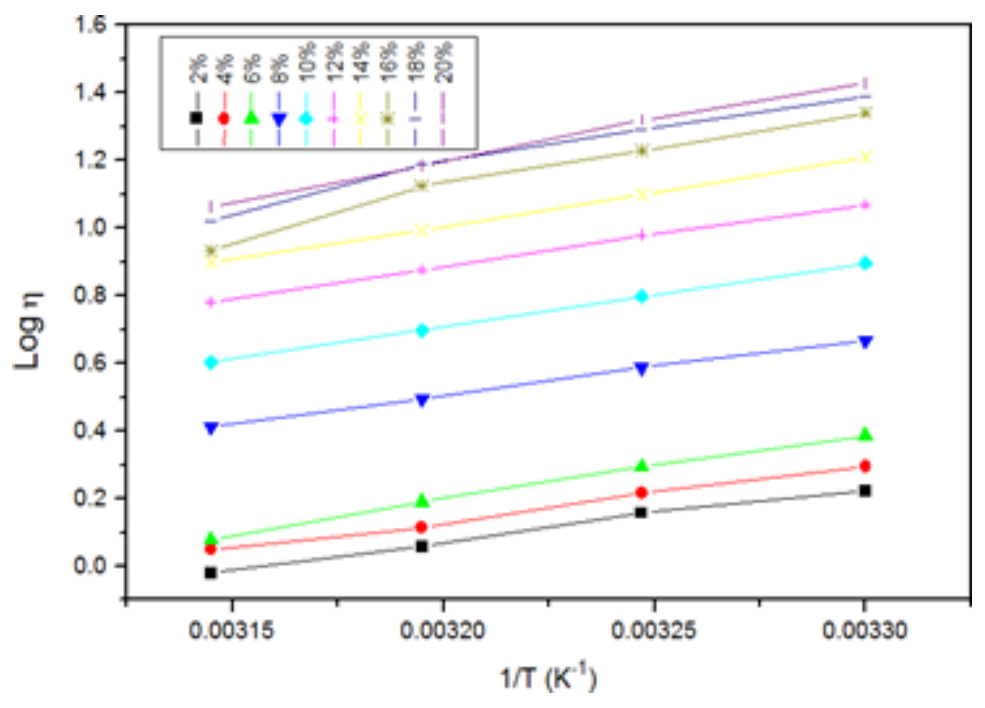

Figure 2 Variation of $1 / \mathrm{T}$ against $\log \eta$

Table 1 Values of relative viscosity for aqueous solutions of Di-Sodium tartrate

\begin{tabular}{|c|c|c|c|c|}
\hline \multirow[t]{2}{*}{ Concentration in (\%) } & \multicolumn{4}{|c|}{ Relative Viscosity (cP) } \\
\hline & 303K & $308 \mathrm{~K}$ & 313K & $318 K$ \\
\hline 2 & 1.25 & 1.17 & 1.06 & 0.98 \\
\hline 4 & 1.34 & 1.24 & 1.12 & 1.05 \\
\hline 6 & 1.47 & 1.34 & 1.21 & 1.08 \\
\hline 8 & 1.95 & 1.8 & 1.64 & 1.51 \\
\hline 10 & 2.45 & 2.22 & 2.01 & 1.83 \\
\hline 12 & 2.91 & 2.66 & 2.4 & 2.18 \\
\hline 14 & 3.35 & 3 & 2.7 & 2.46 \\
\hline 16 & 3.82 & 3.42 & 3.08 & 2.54 \\
\hline 18 & 4.01 & 3.64 & 3.28 & 2.78 \\
\hline 20 & 4.17 & 3.74 & 3.26 & 2.89 \\
\hline
\end{tabular}

The activation energy (Q) is given by the slope of these curves. The variation of activation energy $Q$ with concentration (C) is given in Figure 3.

This graph shows the linear variation of activation energy with an increase in concentration. The activation energy increases with an increase in concentration. The activation energy is minimum at $2 \%$ concentration and maximum at $20 \%$ concentration. From this, it may be concluded that more energy is required to move the molecules inside the structure with an increase in concentration ${ }^{[5]}$. 


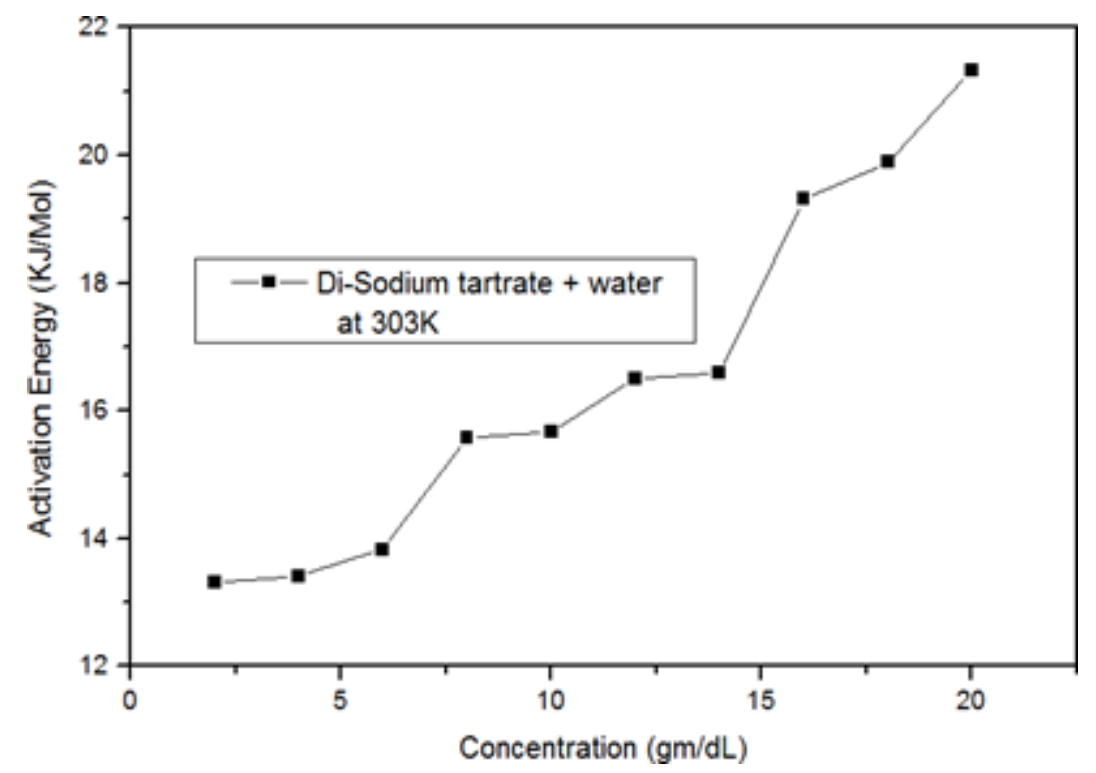

Figure 3 Variation of Activation energy against Concentration

\subsection{Density studies}

The density results show a uniform increase with the increase in the concentration of aqueous solutions of Di-sodium tartrate at $303 \mathrm{~K}$ [Figure 4]. In the lower concentration range (2\%), the density is the minimum for Di-sodium tartrate with a value of $1006.22 \mathrm{~kg} / \mathrm{m}^{3}$. The density of Di-sodium tartrate is the maximum with a value of $1097.89 \mathrm{~kg} / \mathrm{m}^{3}$ at a higher concentration range $(20 \%)$.

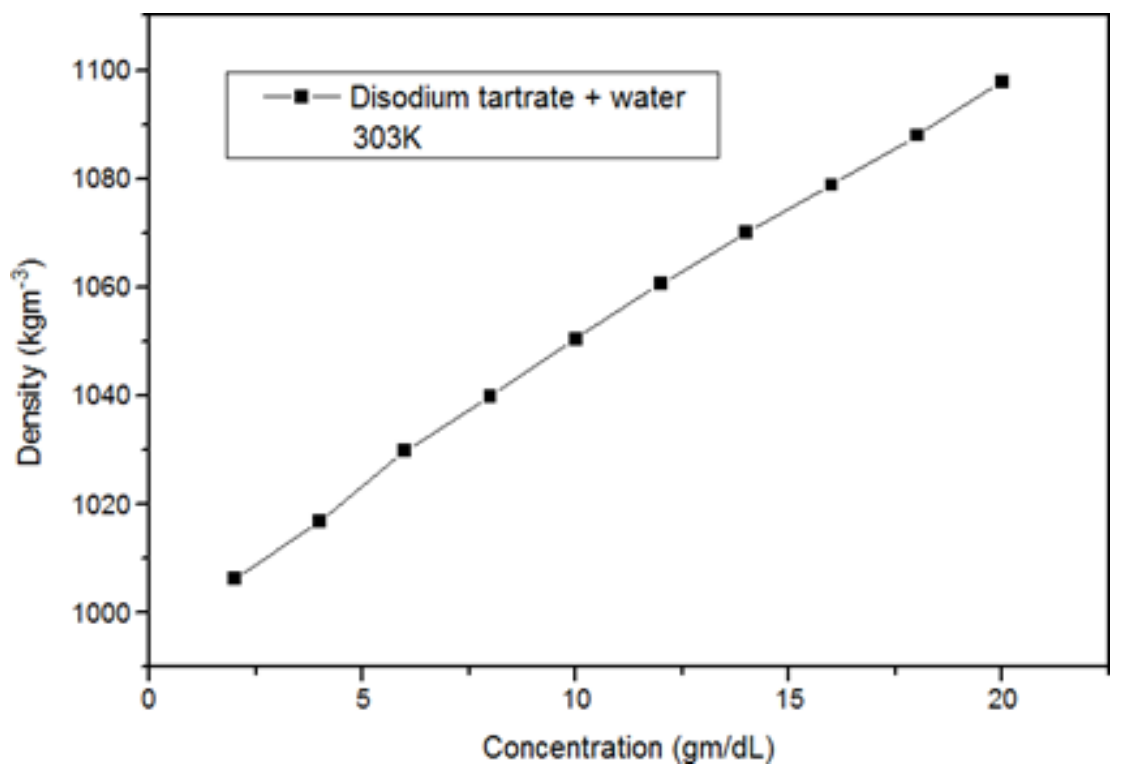

Figure 4 Variation of Density against Concentration

\section{Ultrasonic studies}

\subsection{Theory of sound propagation}

Propagation of sound waves in a medium may be treated as a series of compressions and rarefactions travelling along the direction of propagation such that the molecular planes of the medium are displaced from their mean position. Propagation of ultrasound in a medium is concentration-dependent. Ultrasonic velocity and various parameters are calculated for various concentrations $[2 \%, 4 \%, 6 \%, 8 \%, 10 \%, 12 \%, 14 \%, 16 \%, 18 \%$ and $20 \%]$ of Disodium tartrate in water. 
The displacement $(\xi)$ and velocity of $(C)$ of the waves are related by the wave equation.

$\frac{\delta^{2} \xi}{\delta x^{2}}=\frac{\delta^{2} \xi}{c^{2}} \frac{1}{\delta t^{2}}$

It is assumed here that the compressions are both reversible and adiabatic. The wave equations may be rewritten using the isentropic compressibility $\left(\beta_{\mathrm{s}}\right)$ and the density $(\mathrm{d})$ of the medium as

$\frac{\delta^{2} \xi}{\delta x^{2}}=\beta_{S} d \frac{\delta^{2} \xi}{\delta t^{2}}$

Comparing (a) and (b) it is evident

That $c^{2}=\left(\beta_{s} d\right)^{-1}$ Thus in the limit of above assumptions, the velocity of the sound waves depends on $\beta$ and $\mathrm{d}$.

Hence $\beta=\frac{1}{c^{2} d}$

\subsection{Ultrasonic velocity}

The ultrasonic velocity can be calculated using the formula [ $\mathrm{U}=\mathrm{N} \times \lambda]$. In Water, the ultrasonic velocity increases with increase in concentration of Disodium tartrate and it is shown in Fig. 5. The Ultrasonic velocity is minimum for $2 \%$ and maximum for $20 \%$. The linear increase of velocity with concentration is clearly seen in Disodium tartrate [Figure 5]. Molecular association is thus responsible for the observed increase of ultrasonic velocity in this mixture.

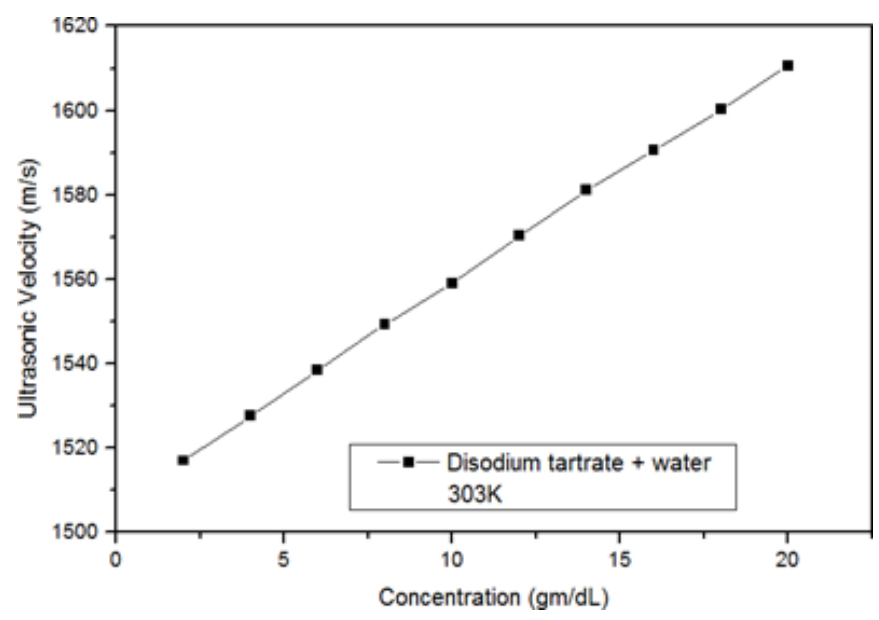

Figure 5 Variation of Ultrasonic Velocity against Concentration

\subsection{Refractive index studies}

Refractive index studies are performed for Disodium tartrate in water in the concentration range of $2 \%$ to 20 $\%$ at 303K. Figure 6 shows the variation of the refractive index against concentration for aqueous solutions of Disodium tartrate at $303 \mathrm{~K}$. In the lower concentration range $(2 \%)$, the refractive index is the minimum for Disodium tartrate in water (1.336). But it is noticed that the refractive index of Disodium tartrate in water is maximum at a higher concentration range $(20 \%)$ with a value of 1.358 . In general, it is observed that the refractive index increases with the increase in concentration. 


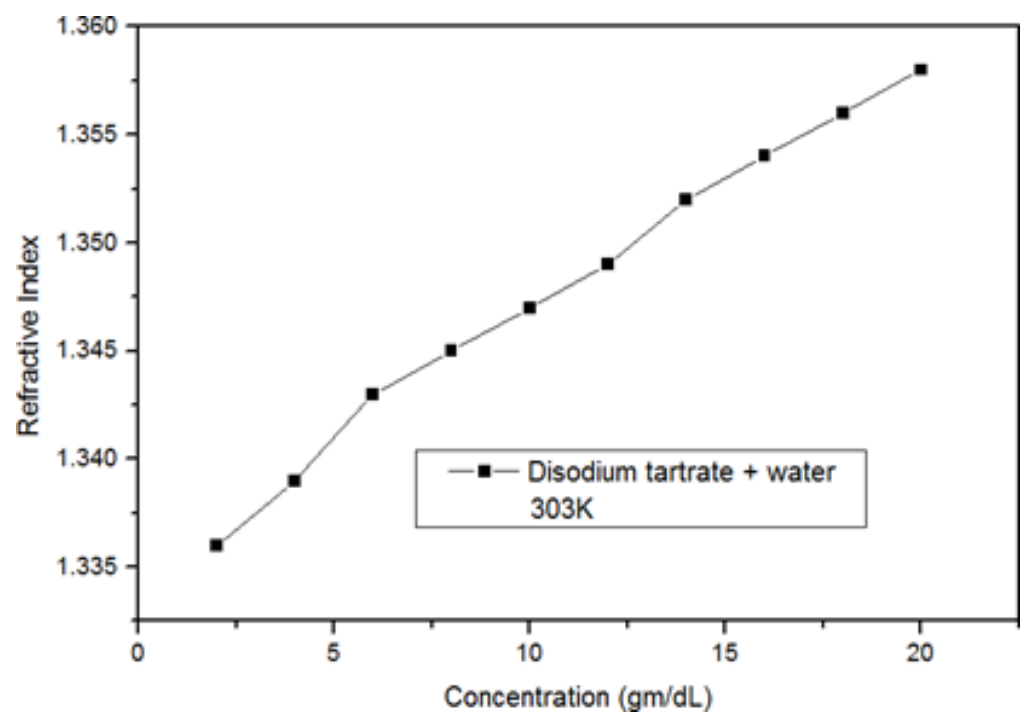

Figure 6 Variation of Refractive Index against Concentration

\section{Molecular interaction parameters}

\subsection{Adiabatic compressibility}

The adiabatic compressibility is calculated using the equation of Newton and Laplace. The structural change in the molecules takes place due to the existence of an electrostatic field between interacting molecules. This structural arrangement or orientation may affect the adiabatic compressibility, which is defined as $\beta=1 / \mathrm{U}^{2} \mathrm{C} \mathrm{m}^{2} / \mathrm{N}$. The compressibility factor is a measure of intermolecular attraction leads to structural particles, which may also decrease the compressibility $[\beta]$ value. The change in the compressibility value in liquids may be ascribed to either the variation in electrostriction or the strength of intermolecular attraction.

$$
\beta_{a d}=\frac{1}{U^{2} d} m^{2} / N
$$

Where, $\beta$ is the Adiabatic compressibility, $U$ is a Ultrasonic velocity, $d$ is the density of the solution.

Normally a decrease in adiabatic compressibility indicates closed packing and decreased ionic repulsion. In the present study, the adiabatic compressibility for an aqueous solution of Disodium tartrate [Figure 7] decreases as the concentration increases [Table 2].

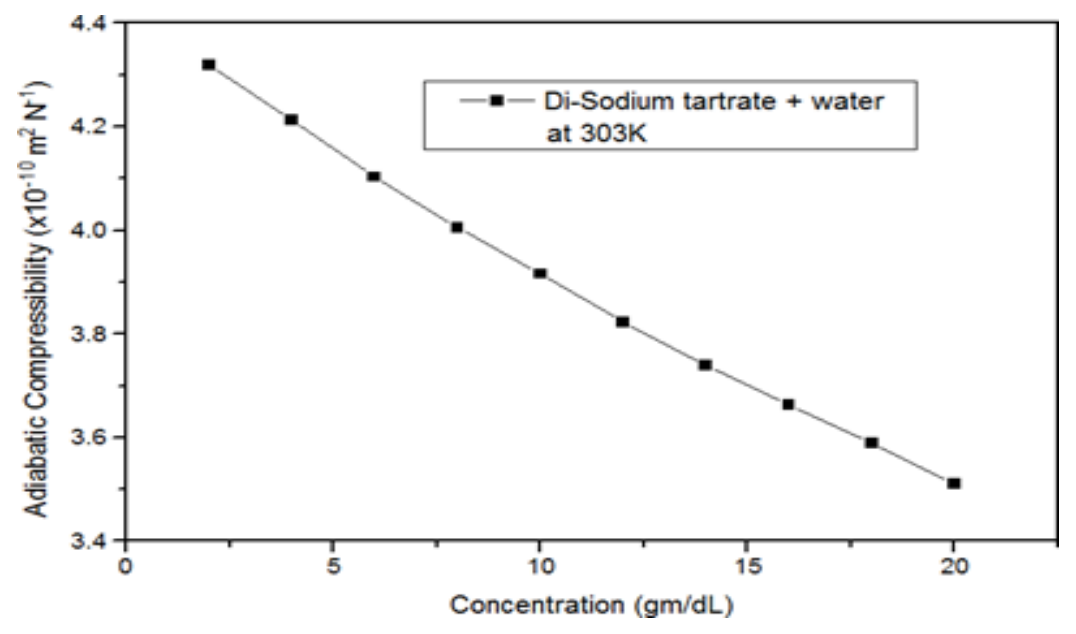

Figure 7 Variation of Adiabatic Compressibility against Concentration 
This indicates that the molecules are closely packed in the solution. The adiabatic compressibility is a maximum of $2 \%$ and minimum for $20 \%$. The adiabatic compressibility shows an inverse behavior as compared to ultrasonic velocity. This indicates that there is a significant interaction between solute and solvent molecules. It can be taken as an indication for the formation of complexes [6].

\subsection{Inter molecular free length}

The increase in free length signifies weak molecular interaction or due to repulsion and an increase in free length signifies specific interaction. The deviation of free length with the increase in ultrasonic velocity indicates the presence of significant interaction between solute and solvent molecules due to which the structural arrangement in the neighborhood of constituent ions is considerably affected.

The intermolecular free length, can be calculated using the formula

$\mathrm{L}_{\mathrm{f}}=\mathrm{k} \beta_{\mathrm{ad}}{ }^{1 / 2} \mathrm{~m}$

Where, $\mathrm{Lf}$ is the Intermolecular free length, $\mathrm{K}$ is Jacobson's constant $\left[\mathrm{K}=(93.875+0.375 \mathrm{~T}) \times 10^{-8}\right]$

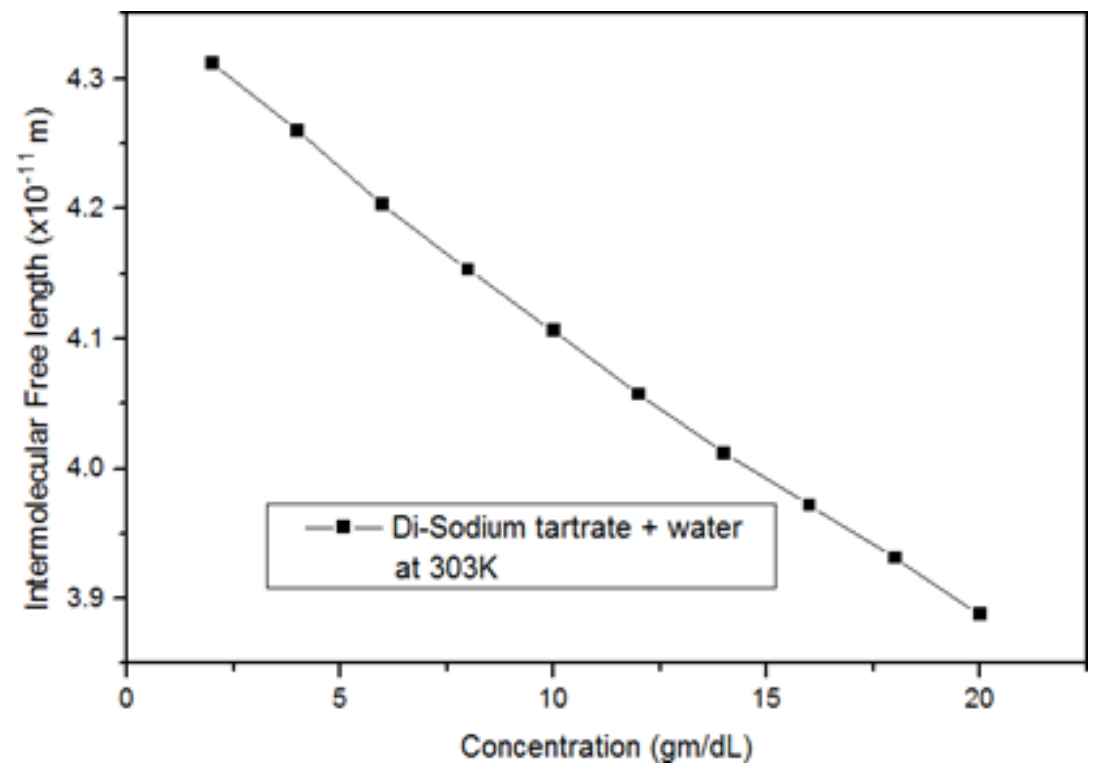

Figure 8 Variation of Intermolecular Free length against Concentration

In the present system, the intermolecular free length for aqueous solutions of Disodium tartrate decreases with the increase in concentration as shown in Figure 8.

The intermolecular free length is maximum for $2 \%$ and a minimum of $20 \%$. In general, for aqueous solutions of Disodium tartrate, at lower concentration range the molecules exhibit weak interaction due to dominant repulsive force and at higher concentration, there is a specific interaction among the molecules. In view of greater forces of interaction between solute and solvent molecules forming hydrogen bonding, there will be a decrease in free length in the mixture $[8,9]$.

The decrease in free length occurs after mixing results in an increase in ultrasonic velocity. This is also in accordance with the expected increase in compressibility following an increase in ultrasonic velocity thereby showing strong intermolecular interactions. Similar results were also reported in few other liquid mixtures[10,11, 13]. 
Table 2 Various Molecular interaction Parameters of aqueous solutions of Disodium tartrate at 303K

\begin{tabular}{|c|c|c|c|c|c|}
\hline $\begin{array}{l}\text { Concentration in } \\
\%\end{array}$ & $\begin{array}{l}\text { Adiabatic } \\
\text { compressibility } \\
\left(\mathbf{x 1 0}^{-10} \mathbf{m}^{2} \mathbf{N}^{-1}\right)\end{array}$ & $\begin{array}{l}\text { Intermolecular } \\
\text { free length } \\
\left(\times 10^{-11} \mathbf{m}\right)\end{array}$ & $\begin{array}{l}\text { Free Volume } \\
\left(\times 10^{-8}\right. \\
\left.\mathrm{m}^{3} \mathrm{~mol}^{-1}\right)\end{array}$ & $\begin{array}{l}\text { Classical Absorption } \\
\text { co- efficient } \\
\left(\times 10^{-14} \mathbf{m}^{-1} \mathbf{s}^{-2}\right)\end{array}$ & $\begin{array}{l}\text { Viscous Relaxation } \\
\text { time } \\
\left({\left.\mathrm{x} 10^{-12} \mathrm{~S}\right)}^{-1}\right.\end{array}$ \\
\hline 2 & 4.3185 & 4.3120 & 1.1851 & 0.9355 & 0.7197 \\
\hline 4 & 4.2140 & 4.2596 & 1.1082 & 0.9718 & 0.7529 \\
\hline 6 & 4.1029 & 4.2030 & 1.0007 & 1.0307 & 0.8041 \\
\hline 8 & 4.0057 & 4.1530 & 0.6791 & 1.3255 & 1.0415 \\
\hline 10 & 3.9159 & 4.1061 & 0.4992 & 1.6178 & 1.2792 \\
\hline 12 & 3.8232 & 4.0572 & 0.3996 & 1.8626 & 1.4834 \\
\hline 14 & 3.7386 & 4.0121 & 0.3348 & 2.0826 & 1.6699 \\
\hline 16 & 3.6638 & 3.9718 & 0.2841 & 2.3134 & 1.8661 \\
\hline 18 & 3.5895 & 3.9313 & 0.2729 & 2.3649 & 1.9192 \\
\hline 20 & 3.5110 & 3.8881 & 0.2658 & 2.3899 & 1.9521 \\
\hline
\end{tabular}

Table 3 Various Molecular interaction Parameters of aqueous solutions of Disodium tartrate at 303K

\begin{tabular}{|c|c|c|c|c|c|}
\hline $\begin{array}{l}\text { Concentration } \\
\text { in } \%\end{array}$ & $\begin{array}{l}\text { Specific } \\
\text { Acoustical } \\
\text { Impedance } \\
\left(\times 10^{6} \mathrm{Kg} \mathrm{m}^{-2} \mathrm{~S}^{-1}\right)\end{array}$ & $\begin{array}{l}\text { Internal pressure } \\
\left(\times 10^{9} \mathrm{~atm}\right)\end{array}$ & $\begin{array}{l}\text { Relative } \\
\text { association }\end{array}$ & $\begin{array}{l}\text { Rao's constant } \\
\left(\begin{array}{ll}\mathrm{x} & 10^{-3}\left[\mathrm{~m}^{3} \mathrm{~mol}^{-1}\right] \\
{\left[\mathrm{ms}^{-1}\right]^{1 / 3}}\end{array}\right)\end{array}$ & $\begin{array}{l}\text { Wada's constant } \\
\left(\times 1^{3} \mathrm{~m}^{3} \mathrm{~mol}^{-1}\right. \\
\left.\left[\mathrm{Nm}^{-2}\right]^{1 / 7}\right)\end{array}$ \\
\hline & 1.5264 & 2.8510 & 1.0024 & 0.2042 & 0.4018 \\
\hline 4 & 1.5534 & 2.9012 & 1.0107 & 0.2062 & 0.4149 \\
\hline 6 & 1.5842 & 2.9920 & 1.0212 & 0.2077 & 0.4292 \\
\hline 8 & 1.6112 & 3.3879 & 1.0289 & 0.2097 & 0.4424 \\
\hline 10 & 1.6378 & 3.7372 & 1.0371 & 0.2116 & 0.4559 \\
\hline 12 & 1.6656 & 4.0067 & 1.0447 & 0.2135 & 0.4696 \\
\hline 14 & 1.6917 & 4.2290 & 1.0515 & 0.2156 & 0.4830 \\
\hline 16 & 1.7159 & 4.4442 & 1.0581 & 0.2177 & 0.4962 \\
\hline 18 & 1.7409 & 4.4832 & 1.0649 & 0.2197 & 0.5097 \\
\hline 20 & 1.7683 & 4.5037 & 1.0724 & 0.2215 & 0.5239 \\
\hline
\end{tabular}

\subsection{Free volume}

Free volume refers to the void space between the molecules, i.e., the volume present as holes because of irregular packing of the molecules. It may be defined as the average volume in which the central molecule can move inside the hypothetical cell freely without affected by the repulsion of surrounding molecules. The free volume is determined by using the formula

$\mathrm{V}_{\mathrm{f}}=\left[\mathrm{M}_{\mathrm{eff}} \mathrm{U} / \mathrm{K \eta}\right]^{3 / 2} \mathrm{~m}^{3} \mathrm{~mol}^{-1}$

Where, $\mathrm{Meff}_{\text {is }}$ the effective molecular weight, $\mathrm{U}$ is an Ultrasonic velocity, $\mathrm{K}$ is a constant $\left(4.28 \times 10^{9}\right)$ which is independent of the nature of the liquid, $\eta$ is a relative viscosity of the solution. 
The above formula suggests that the free volume depends on the ultrasonic velocity directly and viscosity inversely. The free volume plot follows a reverse trend of viscosity. Therefore, viscosity rather than velocity determines the free volume of our system. Since the viscosity of the tartrate is varied due to molecular interaction or tartrate solvation i.e., due to swelling of the tartrate, the same parameters are influencing the free volume also. The free volume reduces when the internal pressure increases.

In the present case, free volume for aqueous solutions of Disodium tartrate is maximum at $2 \%$ and minimum at $20 \%$ [Figure 9]. The free volume gradually decreases from $2 \%-20 \%$ concentration ranges. The reverse trend of free volume with internal pressure is clearly shown in Figure 10 and Table $2 \&$ Table 3.

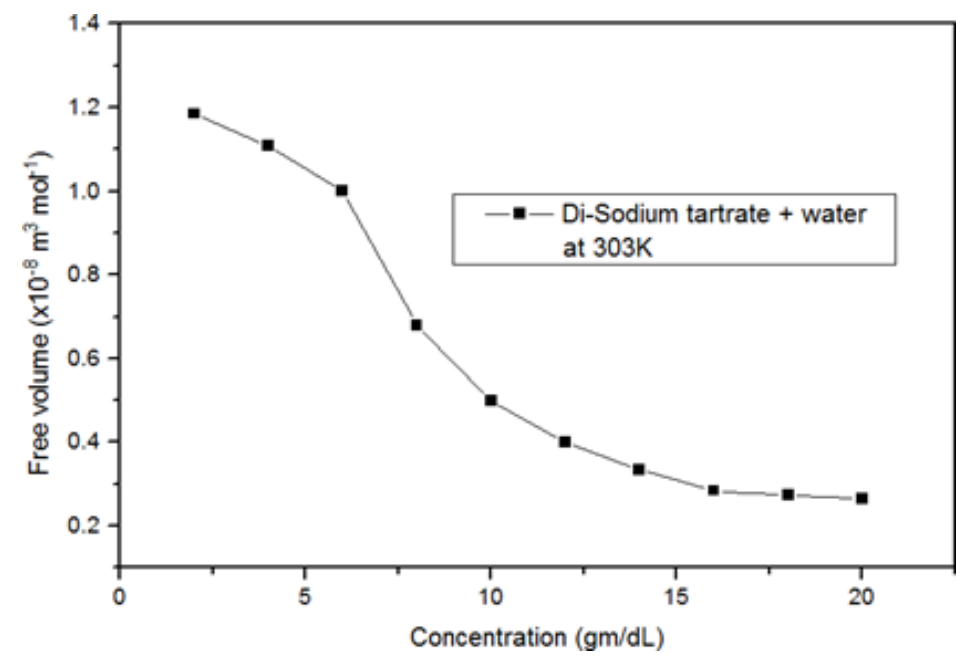

Figure 9 Variation of Free Volume against Concentration

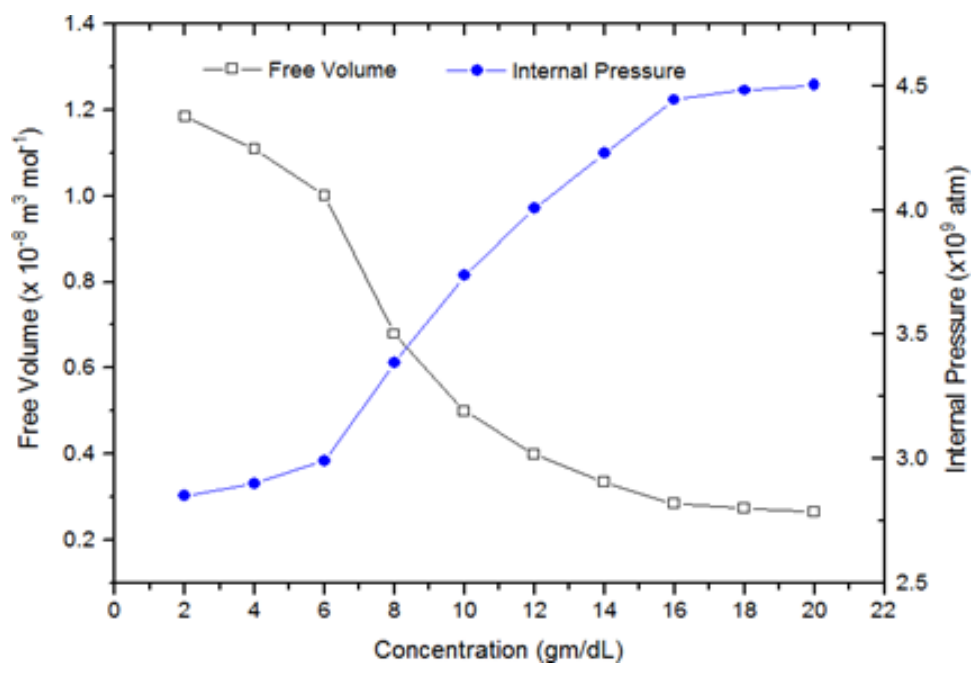

Figure 10 Comparison of Free Volume and Internal Pressure against Concentration

\subsection{Classical absorption coefficient}

The formula for finding the classical absorption coefficient is given below

$$
\frac{\alpha}{f^{2}}=\frac{8 \pi^{2} \eta}{3 d U^{3}} m^{-1} \sec ^{-2}
$$

Where, $\alpha$ is a Absorption coefficient, $\eta$ is the Relative viscosity of the solution, $U$ is Ultrasonic velocity of the solution, $d$ is the Density of the solution. 
In the present system, the classical absorption coefficient for aqueous solutions of Disodium tartrate increases gradually with the increase in concentration as shown in Figure 11. The classical absorption coefficient is minimum for $2 \%$ and maximum for $20 \%$.

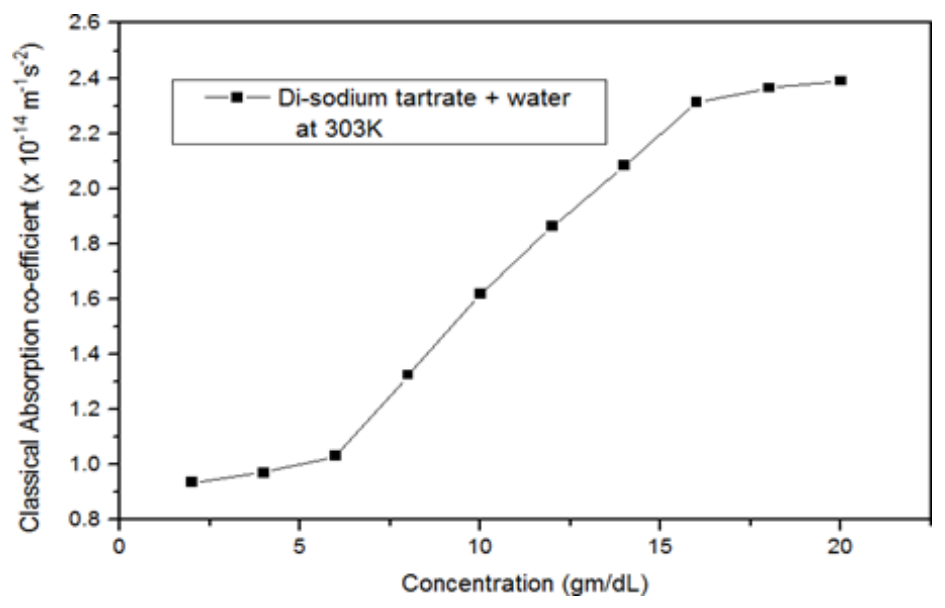

Figure 11 Variation of Classical Absorption Coefficient against Concentration

\subsection{Viscous relaxation time}

Relaxation time was calculated using the formula

$$
\tau=\frac{4 \eta}{3 d U^{2}} \sec
$$

where, $\tau$ is the Relaxation time, $\eta$ is a Viscosity of the solution, $d$ is a density of the solution, $U$ is a Ultrasonic velocity of the solution

From the graph [Figure 12], we see that the viscous relaxation time increases drastically. Aqueous solutions of Disodium tartrate at $303 \mathrm{~K}$. The relaxation time for Disodium tartrate in Water is minimum in lower concentration and maximum at higher concentration range. The relaxation time increases with an increase in concentration throughout the system. The increase in relaxation time confirms the presence of intermolecular interaction.

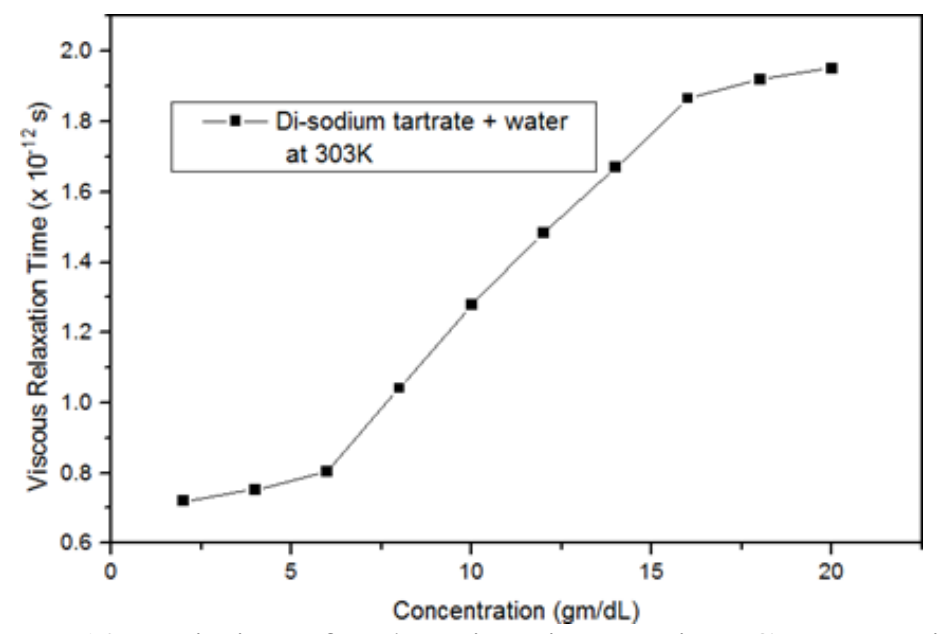

Figure 12 Variation of Relaxation time against Concentration

\subsection{Specific acoustical impedance}

Acoustical Impedance is calculated using the formula 


\section{$\mathrm{Z}=\mathrm{u} \rho \mathrm{Kg} \mathrm{m}^{-2} \mathrm{~S}^{-1}$}

where, $\mathrm{Z}$ is a Acoustical Impedance, $\rho$ is the Density of the solution, $\mathrm{u}$ is a Ultrasonic velocity of the solution.

The acoustic impedance (Z) of a material is defined as the product of its density $(\rho)$ and Ultrasonic velocity (u). Acoustic impedance is important in the determination of acoustic transmission and reflection at the boundary of two materials having different acoustic impedances, the design of ultrasonic transducers, and assessing absorption of sound in a medium.

From the graph [Figure 13] and Table 3, we see that the Specific Acoustical Impedance increases drastically for aqueous solutions of Disodium tartrate. The specific acoustical impedance for aqueous solutions of Disodium tartrate is minimum at lower concentration and maximum at higher concentration. The specific acoustical impedance increases with an increase in concentration throughout the system. This also indicates significant interactions in the solute and solvent.

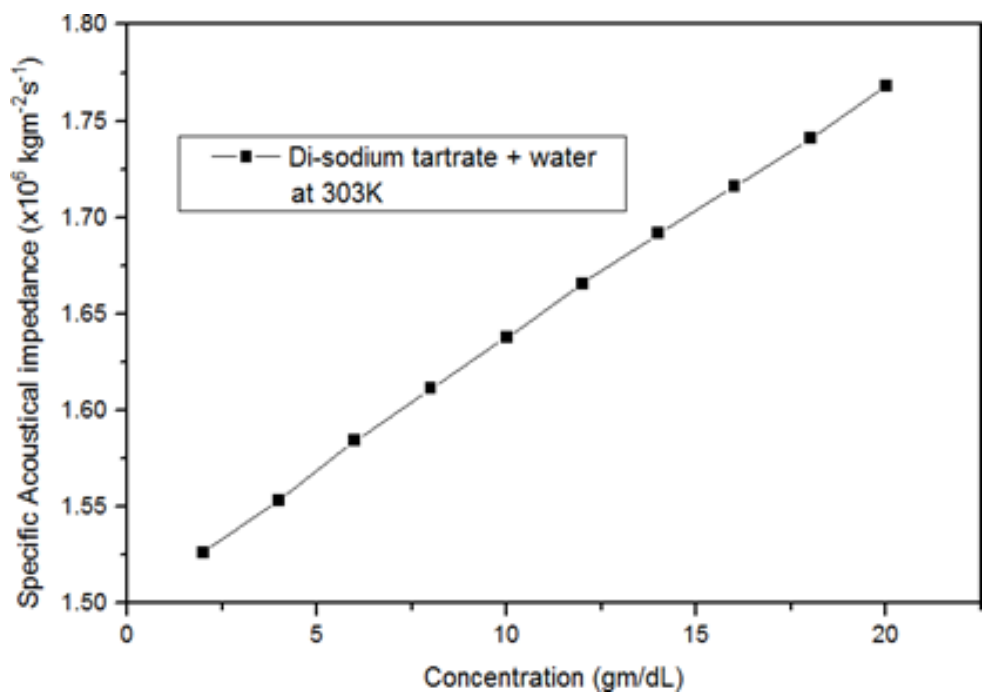

Figure 13 Variation of Specific Acoustical Impedance against Concentration

\subsection{Internal pressure}

Internal pressure is a measure of the resultant attractive and repulsive forces between the interacting components in the mixture.

A new general formula based on dimensional analysis to evaluate internal pressure using ultrasonic velocity is given below.

$$
\pi=b R T \frac{[K \eta]^{1 / 2}}{[U]^{1 / 2}} \frac{d^{2 / 3}}{M^{7 / 6}} a t m
$$

Where, $\pi$ is the internal pressure, $\mathrm{b}$ is the packing factor [assumed as 2 for liquid systems and 1.78 for Polymer solutions], $\mathrm{R}$ is the Universal gas constant, $\mathrm{T}$ is a temperature in Kelvin, $\mathrm{K}$ is a constant of value $4.28 \mathrm{X} 10^{9}, \eta$ is a viscosity in poise, $U$ is a ultrasonic velocity of the solution, $M$ is the effective molecular weight of the solution and $d$ is the density of the solution.

Internal pressure shows a reverse trend to that of free volume as expected, which suggests that the components prevent closed packing of the polymer chains, reducing the molecular cohesion [polymer - polymer interactions and polymer solvent interaction].

The variation of internal pressure with a concentration of aqueous solutions of Disodium tartrate is shown in Figure 14. This study shows that there is an increase in the internal pressure of aqueous solutions of Disodium tartrate with concentration. The internal pressure is minimum at lower (2\%) concentration with a value of $2.8510 \times 10^{9}$ atm and maximum at higher concentration (20\%) with a value of $4.5037 \times 10^{9} \mathrm{~atm}$. 


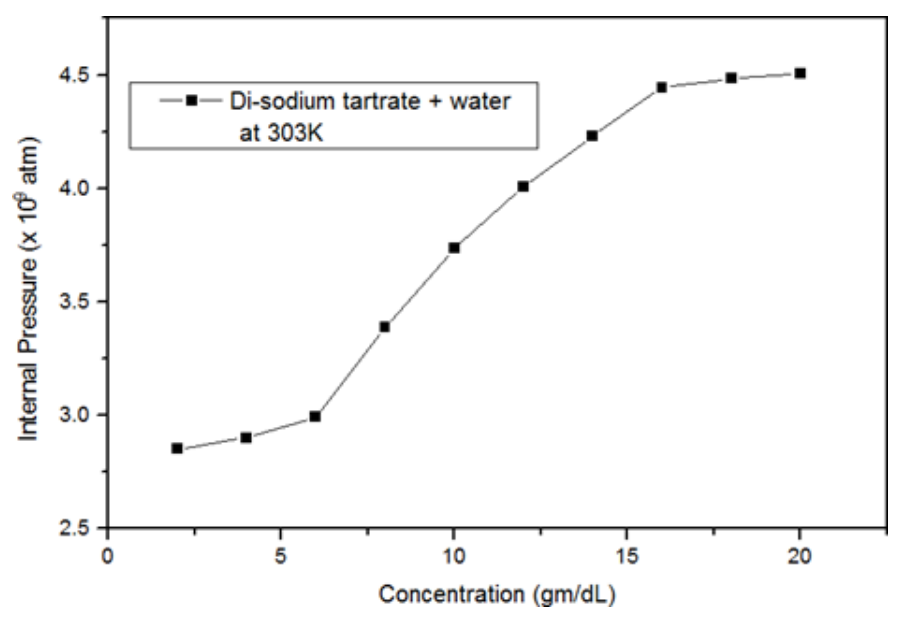

Figure 14 Variation of Internal Pressure against Concentration

\subsection{Relative association}

Relative Association is calculated using the formula

$R A=\left(\rho / \rho_{0}\right)\left(U_{0} / U\right)^{1 / 3}$

Where, $\rho$ is the density of liquid mixture, $\rho_{0}$ is the density of solvent, $\mathrm{U}$ is a Ultrasonic velocity of liquid mixture, $\mathrm{U}_{0}$ is a Ultrasonic velocity of pure solvent.

From the graph [Figure 15], we see that the Relative Association increases drastically for aqueous solutions of Disodium tartrate. The relative association is influenced by two factors (i) breaking up of the associated solvent molecules on the addition of solute in it and (ii) the salvation of solute molecules. The former leads to a decrease and later leads to an increase of relative association.

Generally, the values of (RA) increase with the increase in solute concentration showing significant ion-solvent interaction which increases with an increase in solute concentration [11-13]. In the present system, the variation of Relative Association shows nonlinear variation. The Relative association for Disodium tartrate in Water is minimum at $2 \%$ concentration and maximum at $20 \%$ concentration[14].

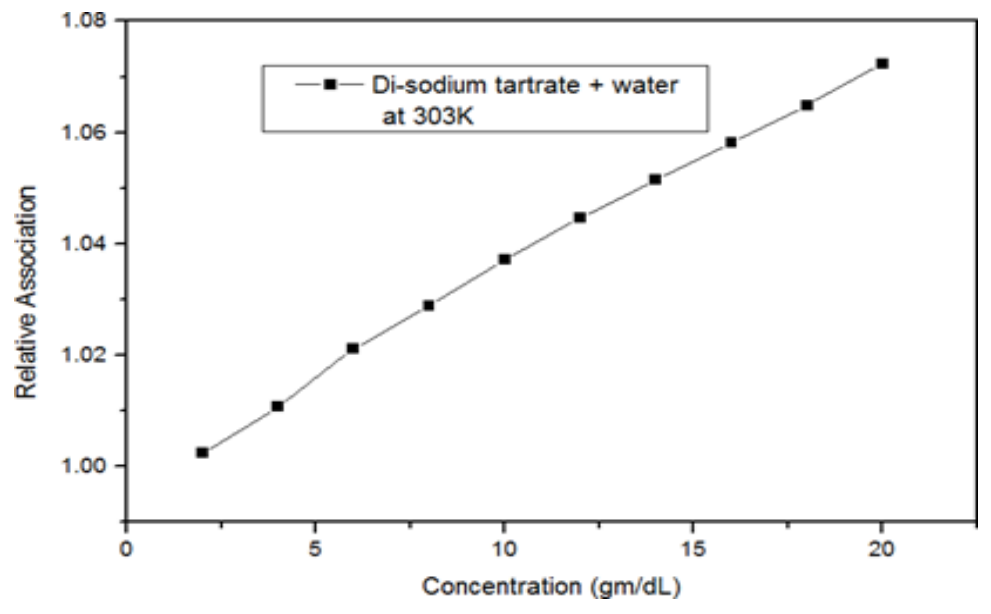

Figure 15 Variation of Relative Association against Concentration

\subsection{Rao'sconstant}

Rao's Constant is calculated using the formula

$\mathrm{R}=\left(\mathrm{M}_{\mathrm{eff}} / \rho\right) \mathrm{U}^{1 / 3}$ 
Where, $\mathrm{M}_{\mathrm{eff}}$ is a Molecular weight of the solution, $\rho$ - is the Density of the solution, U- is the Ultrasonic Velocity of the solution.

From the graph [Figure 16], we see that the Rao's Constant increases linearly in aqueous solutions of Disodium tartrate at $303 \mathrm{~K}$. The Rao's Constant for aqueous solutions of Disodium tartrate is minimum at lower concentration and maximum in higher concentration range. Rao's Constant increases with an increase in the concentration throughout the system taken for study.

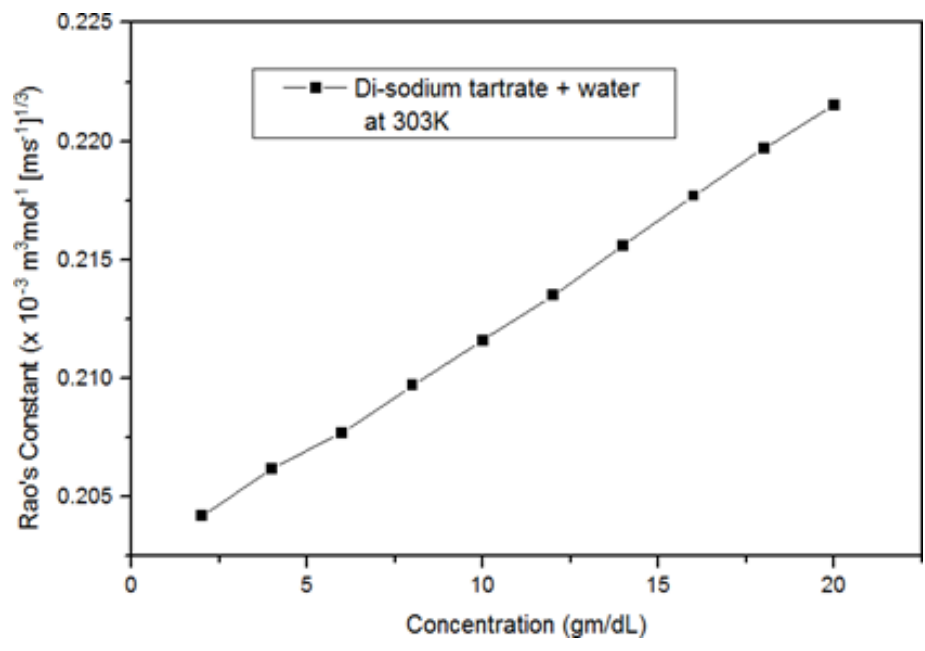

Figure 16 Variation of Rao's Constant against Concentration

\subsection{Wada's constant}

Wada's Constant can be calculated using the formula

$\mathrm{W}=\left(\mathrm{Meff}_{\mathrm{ef}} / \rho\right) \beta^{-1 / 7}$

Where, $\mathrm{M}_{\mathrm{eff}}$ is the molecular weight of the solution, $\rho$ is the density of the solution, $\beta$ is the adiabatic compressibility of the solution.

From the graph [Figure 17], we see that Wada's constant increases linearly with aqueous solutions of Disodium tartrate. Wada's Constant for Disodium tartrate in Water is minimum at lower concentration and maximum at higher concentration range as seen in Table 3. Wada's constant increases with increase in the concentration for the entire system taken for study.

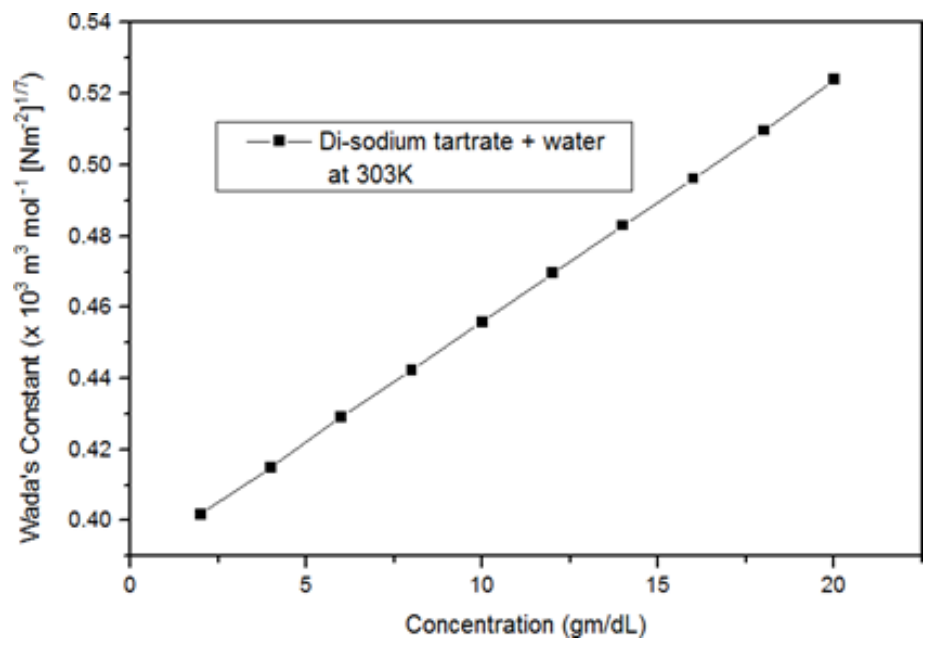

Figure 17 Variation of Wada's Constant against Concentration 


\section{Conclusion}

The results from the viscosity studies shows that the variations in the relativity viscosity from lower to higher concentration level. Similarly, the value of $\ln \eta$ increases with concentration and decreases with decrease in temperature. And also, the activation energy and density results indicate the values increases with the increase in concentration of aqueous solution of Di-sodium tartrate at $303 \mathrm{~K}$. Due to the molecular association, the ultrasonic velocity and refractive index values changes over the increases in concentration range.

In this, the adiabatic compressibility shows an inverse behavior as compared to ultrasonic velocity due to the closely packed molecules in the solution. Similarly, the intermolecular free length value decreases with the increases in concentration. The intermolecular free length is maximum for $2 \%$ and minimum for $20 \%$. The free volume formula suggests that the free volume depends on the ultrasonic velocity directly and viscosity inversely. The free volume reduces when the internal pressure increases.

The classical absorption coefficient and the relaxation time for Disodium tartrate in water is minimum in lower concentration and maximum at higher concentration range. From graph, it was revealed that the specific Acoustical impedance increases drastically for aqueous solutions of Disodium tartrate. It shows that the significant interactions in the solute and solvent. The results from the internal pressure study, shows that the value of $2.8150 \times 10^{9} \mathrm{~atm}$ at lower concentration and $4.5037 \times 10^{9} \mathrm{~atm}$ at higher concentration. The relative association for Disodium tartrate in water is minimum at $2 \%$ concentration and maximum at $20 \%$ concentration. It was noted that the Rao's constant increases with increase in the concentration throughout the system. And also, found that the Wada's constant increases with increase in the concentration for the entire system from the graph.

\section{Compliance with ethical standards}

\section{Acknowledgments}

I wish to thank Department of Physics, SCSVMV University, Kanchipuram for providing the necessary experimental facilities for compilation of the research work.

\section{Disclosure of conflict of interest}

The authors hereby attest to the fact that there is no any conflicting interest of any sort in this study.

\section{References}

[1] Housecroft, C. E.; Sharpe, A. G.Inorganic Chemistry (3rd ed.). 2008; Prentice Hall.

[2] https://science.jrank.org/pages/6698/Tartaric-Acid.html

[3] Lide, David R., Handbook of Chemistry and Physics (87 ed.), Boca Raton, Florida: CRC Press,1998; 3-502.

[4] Moore W.R. and Brown A.M. 'Viscosity-temperature relationships for dilute solutions of cellulose derivatives: Temperature dependence of solution viscosities of ethyl celluslose', Journal of Colloid Science, 1959; 14 (1) 1-12.

[5] Guadalupe Olayo Ma., Guillermo J., Cruz., Salvador Lopez., Juan Morales., Roberto Olayo. “Conductivity and Activation energy in Polymers synthesized by Plasmas of Thiophene”. J. Mex. Chem., Soc. 2010; 54(1):18-23.

[6] Kubendran R., Liakath Ali Khan F., Asghar J., Aravinthraj M. and Udayaseelan J. 'Ultrasonic studies of N, NDimethylformamide with Alkoxyethanols in Carbon tetrachloride at different temperatures' Archives of Applied Science Research, 2011; 3(3):296 -301.

[7] Suhashini Ernest and Kavitha P.'Acoustical and Excess Thermodynamical Parameters of Sesame Oil in Different Organic Solvents', International Journal of Chemical environmental and Pharmaceutical Research, 2011; 2 (2-3): $111-115$.

[8] Asghar J., Liakath Ali Khan F. and Subramani K. 'Thermodynamic studies of molecular interactions in ternary liquid mixtures at various temperatures', Rasaya J Chem,2010; 3(4):697-704.

[9] Jayakumar S., Karunanithi S. and Kannappan V. 'Ultrasonic velocity and other acoustical parameters in binary mixtures of O-cresol, P-cresol, Ochlorophenol and P- chlorophenol with CCl4 at 303.15 K' Ind. J pure \& Appl. Phys. 1996; 34(10):761-763. 
[10] Kannappan A N, Kesavasamy R. and Ponnuswamy V., Molecular Interaction Studies of H-Bonded Complexes of Benzamide in 1,4-Dioxan with Alcohols From Acoustic and Thermodynamic Parameters', Am. J Eng and Appli. Sci., 2008; 1(2):95-99.

[11] Syal V.K., Lal G., Bisht P. and Chauhan S. Ultrasonic measurements of some 1:1 electrolyte in chlorobenzene + methanol mixtures', J. Mol. Liquids.1995; 63:317-328.

[12] Prasad R, Kamal S, Sharma PK, Oelmüller R, Varma A. Root endophyte Piriformosporaindica DSM 11827 alters plant morphology, enhances biomass and antioxidant activity of medicinal plant Bacopa monniera. Journal of Basic Microbiology. 2013 Dec;53(12):1016-24.

[13] Sathish .M., Meenakshi .G 'Ultrasonic study of some Amino Acids in Aqueous Salt Solution in 303.15K' . International Journal of Research in Engineering and Technology 2014 Feb;3 (2):312-317.

[14] G Ramalingam, R Vignesh, C Ragupathi, C Maria Magdalane, K Kaviyarasu, J Kennedy, Electrical and chemical stability of CuS nanofluids for conductivity of water soluble based nanocomposites, Surfaces and Interfaces June 2020; 19:100475. 